\title{
Radio-Nuclear Investigations in Children Aged 16 Years and Younger with Confirmed Urinary Tract Infection: A Cross Sectional Retrospective Study
}

\author{
Reem Al Qutami Al Suwaidi, ${ }^{1, ~ *, ~ S a m ~ H a s s a n ~}{ }^{2}$ \\ ${ }^{1}$ College of Medicine, Mohammad Bin Rashid University for Medicine and Health Sciences, Dubai, United Arab Emirates \\ ${ }^{2}$ Department of Pediatrics, Mediclinic City Hospital, Dubai, United Arab Emirates
}

Email address:

Reem.alqutami@gmail.com (R.Al Q.Al Suwaidi),samhassan@gmail.com (S. Hassan)

${ }^{*}$ Corresponding author

\section{To cite this article:}

Reem Al Qutami Al Suwaidi, Sam Hassan. Radio-Nuclear Investigations in Children Aged 16 Years and Younger with Confirmed Urinary Tract Infection: A Cross Sectional Retrospective Study. American Journal of Pediatrics. Vol. 5, No. 1, 2019, pp. 7-13. doi: 10.11648/j.ajp.20190501.12

Received: December 23, 2018; Accepted: January 29, 2019; Published: February DD, 2019

\begin{abstract}
A retrospective cross-sectional study conducted at Mediclinic City Hospital; a teaching hospital in collaboration with Mohammad Bin Rashid University for Medicine and health sciences in Dubai, United Arab Emirates (UAE). The aim of this study is to ascertain conduction of renal radio nuclear investigations, explicitly Kidney ureter bladder ultrasound scans (KUB US), Micturating cystourethrogram (MCUG), and Dimercaptosuccinicacid (DMSA) in children following Urinary Tract Infection (UTI), based on; age, type of UTI, and recurrence. Medical records of 421 patients aged 0-16 years with UTI were retrospectively studied. Sociodemographic variables were age, gender, type of UTI, and recurrence. Results showed that the most carried out investigation was KUB US (38.2\%) $(\mathrm{n}=161)$. In children aged between 7 months and 3 years, $15 \%(\mathrm{n}=8)$ of them had VUR or renal scarring, while only12\% $(\mathrm{n}=11)$ of children aged $>3$ years showed abnormal MCUG or DMSA regardless of the KUB US results. Interestingly more than half $(56 \%)(n=236)$ of all patients $(n=421)$ with recurrent UTI showed abnormalities by MCUG and or DMSA, while only $12.3 \%(\mathrm{n}=14)$ of children with atypical UTI showed abnormalities. It is concluded that VUR and renal scarring predominantly occurred in patients with recurrent UTI and atypical UTI. Thus, children presenting with recurrent UTI are recommended to undergo MCUG and DMSA after the second recurrent episode rather than waiting for the third UTI incident. This study suggests that children aged 7 months to 3 years post UTI should undergo KUB US, MCUG (if it was a recurring UTI) plus DMSA or MAG3 with post-micturition study instead. Children older than 3 years presenting with recurrent UTI are recommended to undergo DMSA (regardless of the US results) and MAG3 with post-micturition study or MCUG especially if DMSA is abnormal. Keeping in mind, evaluating risk factors like family and past medical history are crucial before conducting any investigation, this is to avoid unnecessary scans, and at the same time implement measures to reduce risks resulting from complicated UTIs.
\end{abstract}

Keywords: Urinary Tract Infection, Children, Pediatrics Nuclear, Radiological, Investigations

\section{Introduction}

Radio-nuclear investigations following UTI in children remains a controversial topic. There is no consensus of when to perform DMSA and MCUG scans. Hence, local and global guidelines are needed.

Urinary tract infection (UTI) is one of the most common bacterial infections encountered in childhood [1]. Although classical UTI is of no serious consequences if detected and treated early, some consequences can be lifelong if the infection is associated with other urinary tract abnormalities such as: VUR, recurrent infections, atypical infections or complicated UTI [2]. Likewise, if not properly detected or treated, consequences such as hypertension, kidney dysfunction, renal scarring, and other complications could be life-threatening [3]. Therefore, a high level of diagnostic 
precision and consistency is crucial specially in the pediatric age group. This observational study will closely focus on the diagnoses and the following up stages of UTI, including conducting investigations to detect consequences or underlying causes of UTI. Renal radio investigations following UTI are usually carried for further diagnostic modalities; such as using MCUG to detect underlying abnormalities that possibly predispose to recurrent UTI and scarring like VUR, or using DMSA for detecting UTI related complications for instance: renal scarring [3]. Renal scarring has tremendously increasing risk in children aged less than 6 months following UTI, recurrent UTI, and in VUR [4- 5].

MCUG and DMSA are invasive radiological procedures that are usually carried out to help avert renal insufficiency after UTI in children. However, the psychological impacts of such invasive procedures, like developing medical fears, reduced rate of health control, and posttraumatic stress reactions [6] can be serious in children. Yet, it is not adequately being considered by physicians and medical practitioners. Moreover, the radiation risk in children exposed to by undergoing these radio investigations is tremendous. It has been proven that radiation exposures from MCUG and DMSA are associated with equivalency of 75 and 50 chest X-rays respectively [3]. Hence, it is essential for physicians and medical practitioners to weigh the benefit to risk ratio. Also, having a proper and consistent strategy is crucial to avoid unnecessary invasive procedures, but at the same time balancing the benefits against the risks in this yet controversial issue.

According to a recent study, ideally, DMSA scans should be intended to perform 3 months after presentation with recurrent UTI and not before. DMSA scans can be reserved for preforming after revealing abnormal MCUG indicating presence of VUR. The study also showed minimal difference between the results of US and DMSA scans among those with recurrent UTI [7]. In contrast, another research proved that the sensitivity of US for detecting renal scarring was $33.3 \%$ in school age children with recurrent UTI, which is considered poor [8]. Hence, this topic is still a controversial subject and has been evolving over the past decades despite many studies. Some studies advice of conducting all investigations at once for certain age groups, while others recommend discretion of investigations depending on age, type of UTI and results of Ultrasound (KUB US).

As far as this study is concerned, this is the first research study that is conducted in the United Arab Emirates (UAE) to evaluate and explore renal radio nuclear investigations. This research was aimed to evaluate renal radio nuclear investigations in pediatric patients following UTI in Mediclinic City Hospital which is a tertiary teaching hospital in Dubai. The objectives were to determine proper appropriate scheme strategies in which different radio imaging investigations can be carried out in relation to; specific pediatric age groups, recurrence, and type of UTI. Our hypothesis claimed; variations will be seen in regards to the investigations conducted and scheme recommendations will differ depending on age, type of UTI, and US results.
Findings of this study may help apprise guideline provision efforts to reduce or add nuclear investigation procedures. Findings may also provoke counteractive or precautionary interventions.

\section{Material and Methods}

A retrospective cross-sectional study, in which all medical records of patients presenting with UTI to $\mathrm{MCH}$, aged 0-16 years were retrospectively viewed and analyzed. Medical records were acquired from $\mathrm{MCH}$, a tertiary hospital located in Dubai, UAE. The analysis took place at Mohammed Bin Rashid University (MBRU), College of Medicine located in Dubai Health Care City, UAE.

All patients presenting to $\mathrm{MCH}$ aged 0-16 years, who have experienced a UTI episode between 1 January to 31 December, 2017 were included in the study. Method of selecting is convenient sampling method. List of patients was obtained from the microbiology lab based on positive urine culture and urine analysis. The study population was divided into three groups with respect to age; first group: 0-6 months, second group: 7 months -3 years, and the third group older than 3 years. The study population was further divided into 3 additional UTI classification groups (Typical UTI, Atypical UTI, Recurrent) within each age group.

A UTI episode was defined as a positive urine culture of more than 100,000 colonies per cubic milliliter and urine analysis. Atypical UTI was defined when the pathogen is any organism other than Escherichia coli. Recurrent UTI was defined as 3 UTI episodes in a 1-year duration [7]. Investigations that were explored in this study were MCUG, DMSA, and KUB US. Renal US was considered abnormal if any of the following were present, thickened bladder wall, atrophied kidney, hydronephrosis, the presence of residual volume, ureteric- pelvic dilatation, renal or bladder calculi and the presence of cysts on adnexa. Abnormal DMSA was considered when the presence of any form of kidney scarring was present. MCUG was defined as either unilateral or bilateral VUR grade 1-4.

The MRN (Medical Record Number) of patients were first obtained from the Information Technology (IT) department in $\mathrm{MCH}$. The list of MRN was based on the 'diagnosis of UTI' documented for insurance forms, which preliminary assessed 100 UTI cases. Most UTI cases were suspected cases and not confirmed. Hence, research was further continued in regards to the MRN list that was obtained from the microbiology lab based on positive urine cultures and urine analysis. The clinical researcher member of whom documented the findings from the HIS (Hospital Information System) had undergone a 2-hour comprehensive training course on how the HIS exactly works. Also, at the time of data documentation, the supervised clinician or his nurse had supervised the researcher member entering the data, this was to examine the accuracy and completeness of data. This study has been approved by the MBRU-IRB (Institutional Review Board) ethical committee and Mediclinic research 
committee. (MBRU-IRB-SRP2018-034).

Data were initially recorded and documented on a Microsoft Excel sheet and then transferred to the Statistical Package for the Social Science software (SPSS) to be analyzed. A time frame sample size from 1 January to 31 December 2017 was used, ending up with 421 subjects. Quantitative variables were presented by percentages and no statistical tests are needed, since this is chiefly a descriptive study. Categorical data such as the prevalence of each type of UTI is deliberated as percentages.

\section{Results}

In this study, a total of 421 medical records of children aged 0-16 years, with confirmed UTI were viewed. 313 patients of which were females $(74.3 \%)$ and 108 males $(25.7 \%)$. The mean $( \pm \mathrm{SD})$ age of patients was $5.1 \pm 4.2$ years. The first age group ( 0 to 6 months old) constituted of 54 patients $(12.8 \%)$, while the second age groups ( 7 months to 3 years) 135 patients $(32 \%)$ and the third group (above 3 years old) 232 patients (55\%), (Figure 1).

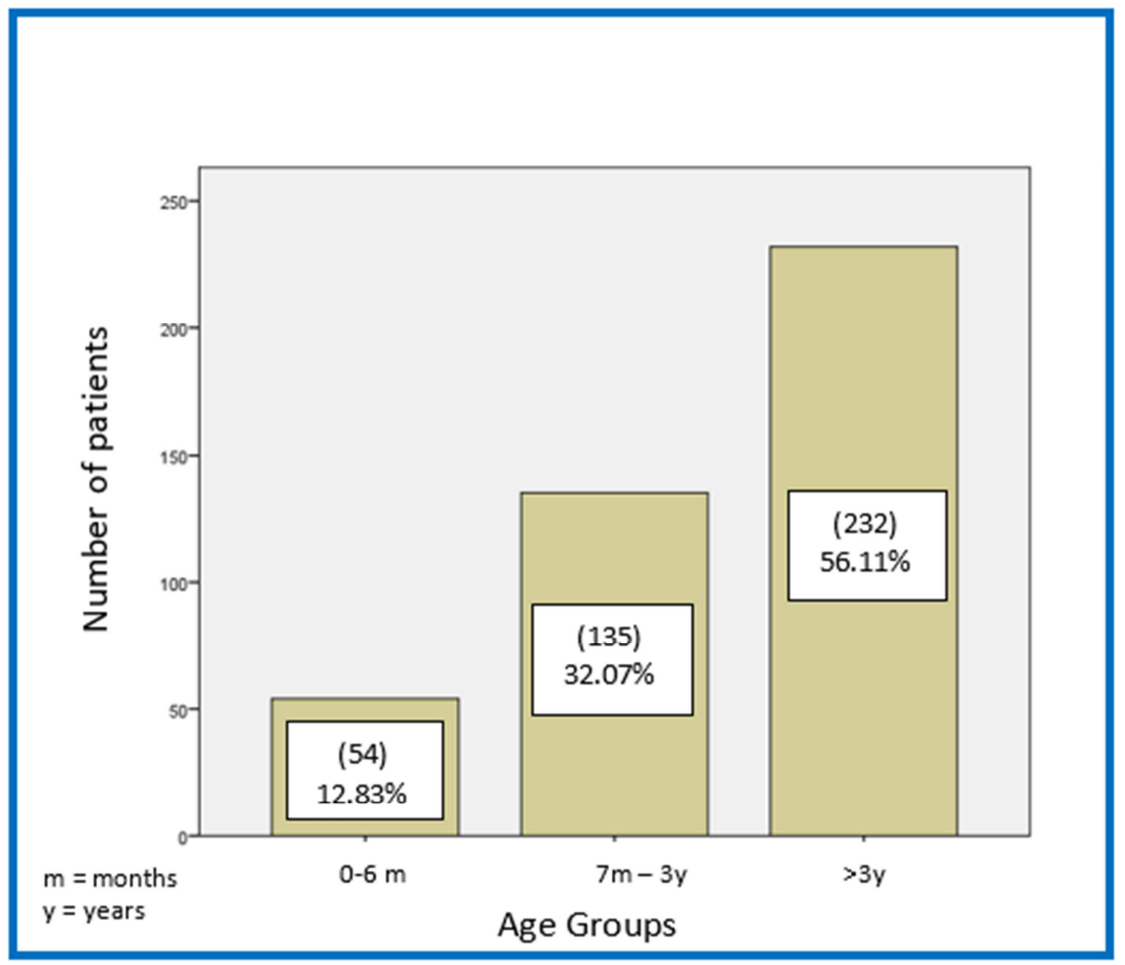

Figure 1. Total number (in brackets) and percentages of patients with UTI in regards to each age group.

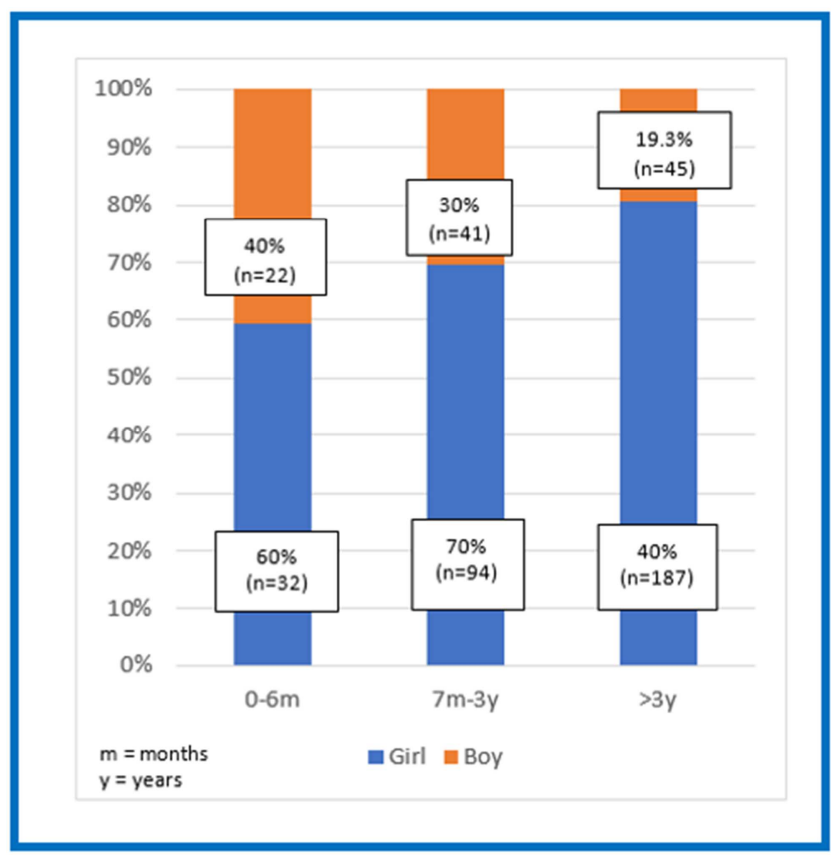

Figure 2. Gender percentages and number (in brackets) per age group.

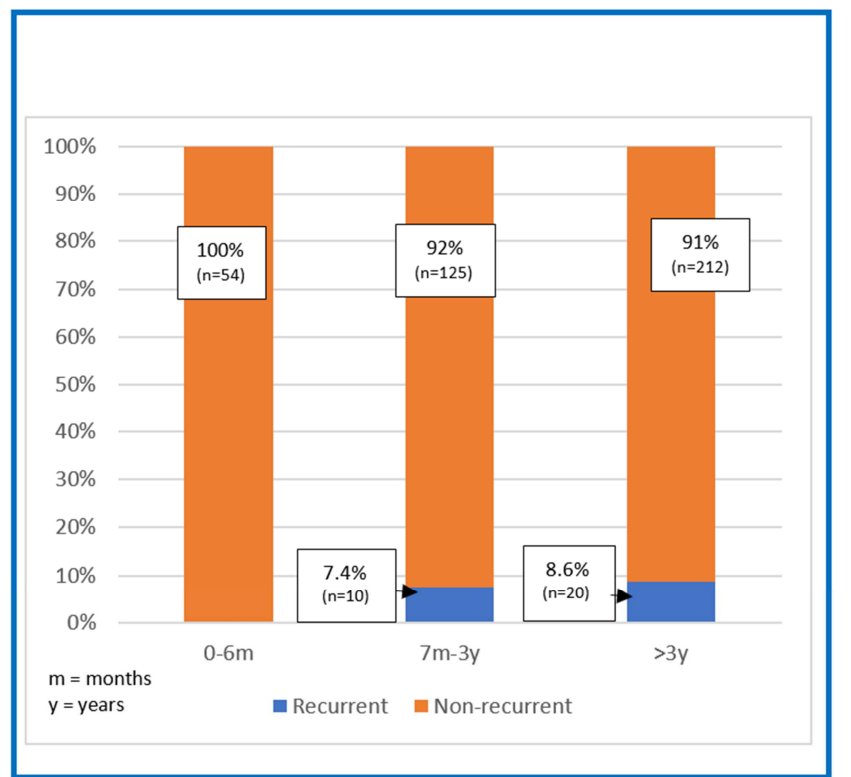

Figure 3. Percent and number (in brackets) of recurrent UTI cases per age group. 
The prevalence of UTI was the highest among the third age group and found mainly in females (Figure 2). Most cases were typical UTI cases (E Coli) $(73.2 \%)$ while the prevalence of atypical UTI was only $26.8 \%(\mathrm{n}=113)$. Out of the atypical cases $47 \%(n=53)$ were aged over 3 years, $38 \%$ $(\mathrm{n}=43)$ aged 7 months to 3 years, and $15 \%(\mathrm{n}=7)$ aged $0-6$ months old. Total number of patients presented with recurrent UTI was $7.2 \%(n=30)$, in which 20 cases $(66.7 \%)$ were above 3 years, and the other 10 cases $(33.3 \%)$ were aged 7 months to 3 years. (Figure 3 )

Renal radio-nuclear investigations were inconsistent in all age groups and the most frequently carried procedure is the KUB US scans. Number of patients underwent KUB US is $161(38.2 \%)$, while 33 patients (5\%) only had MCUG and 6 patients $(1.4 \%)$ underwent DMSA. Abnormal US results were mostly found in the third age group (Figure 6) specifically among males. Among the patients who had KUB US (161 patients), 44 (27\%) had abnormal US results. Eight patients of the 44 had recurrent UTI (18.1\%), and 9 had atypical UTI (20\%). Thirty-three patients had MCUG done. Sixty percent $(\mathrm{n}=20)$ had abnormal results, and $80 \%$ $(n=16 / 20)$ of those children with abnormal MCUG results were females. Forty-five percent $(n=9 / 20)$ of patients with abnormal MCUG results had recurrent episodes, and most of the cases $(80 \%)(n=16 / 20)$ presented with typical UTI. The least conducted investigation was DMSA with 6 patients only $(1.4 \%)$. Most patients had normal DMSA (66\%) and only $33 \%$ had abnormal results. Most patients with abnormal results were in the second and third age groups (Figures 5\&6), all patients were females and all cases were nonrecurring. Fifty percent had atypical UTI (regardless of age).

Only 21 patients in the youngest age group (0-6 months) underwent US (38\%), out of which 5 patients $(23 \%)$ had abnormal results. It is noted that none of these patients had further been investigated by MCUG neither DMSA scans. However, it is not acknowledged if these investigations have been carried out in other centers. (Figure 4).

In the second age group (7 months to 3 years old), only 52 patients (38.5\%) underwent KUB US. Out of those, 38 (73\%) had normal scan results and $14(26 \%)$ had abnormal results. Interestingly, $3 / 4(70 \%)$ of children in this age group who have undergone MCUG with normal US, had abnormal MCUG scans with VUR. Moreover, $100 \%(\mathrm{n}=4)$ of children who have undergone MCUG with abnormal US, showed VUR. On the other hand, only one child underwent DMSA which revealed abnormal results, despite normal US. (Figure 5).

Prevalence of UTI was the highest in the third age group $(n=232)$. Out of 232 patients only $88(37 \%)$ patients had renal US scans, majority were normal $(n=63 / 88)(71 \%)$. Of these patients, only 11 out of 63 underwent MCUG. More than half (6 out of 11), (54\%) showed VUR, despite normal US scans. (Figure 6) Moreover, $66 \%(\mathrm{n}=4)$ among those who have had MCUG done with abnormal US scans showed VUR. Only 1 patient further underwent DMSA with previously abnormal US, which turned abnormal.

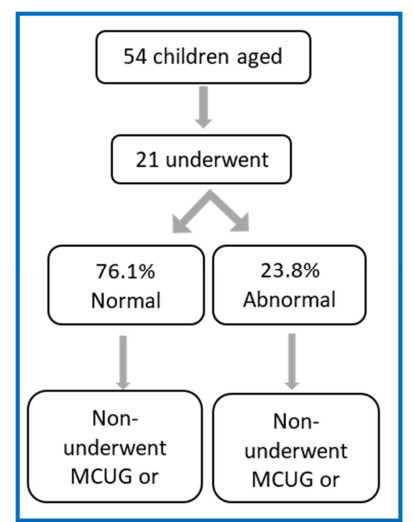

Figure 4. Participants flow chart of the first age group.

The prevalence of recurrent UTI (irrespective of the age) was $7.1 \%(n=30)$. Twenty patients conducted US, 8 of which were abnormal $(40 \%)$. Out of the 30 patients with recurrent UTI, 15 of them (50\%) had MCUG done. VUR was found in $60 \%$ of those patients. Only 1 child underwent DMSA which turned normal. (Figure 5)

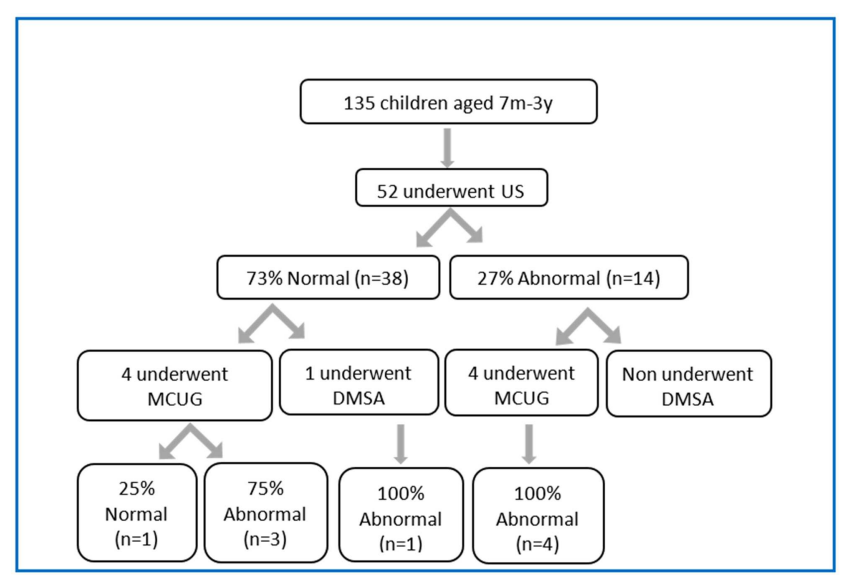

Figure 5. Patients flow chart of the second age group.

Atypical UTI was seen in 113 patients (26\%). Out of these patients only $23(20 \%)$ had underwent US, from which $28 \%$ $(n=9)$ had abnormal scans. Only 6 patients $(5 \%)$ further underwent MCUG, 4 patients (66\%) of them had VUR. Three patients underwent DMSA, and only one (33\%) showed scaring.

\section{Discussion}

The most carried out investigation among all patients (421) was KUB US ( $\mathrm{n}=161)(38.2 \%)$ followed by MCUG $(\mathrm{n}=32)$ $(7.8 \%)$, then DMSA $(\mathrm{n}=6)(1.4 \%)$. None of the subjects aged 03 months underwent MCUG nor DMSA (Figure 4), despite the recommendations of most guidelines. Fifteen percent $(n=20)$ of all patients $(\mathrm{n}=135)$ aged 7 months -3 years, who have undergone KUB US (regardless of the results) had serious abnormalities such as scarring or VUR (Figure 5). Twelve percent $(n=28)$ of all patients $(n=232)$ aged older than 3 years, who have undergone US (regardless of its results) had serious complication whether it was kidney scarring or VUR (Figure 6). 
More interesting is that $56 \%(n=17)$ of all patients $(n=30)$ with recurrent episodes of UTI, had abnormal US and MCUG results (Figure 7). In the group of atypical UTI $(n=113), 12.3 \%(n=14)$ of patients had abnormal US, MCUG, and DMSA (Figure 8).

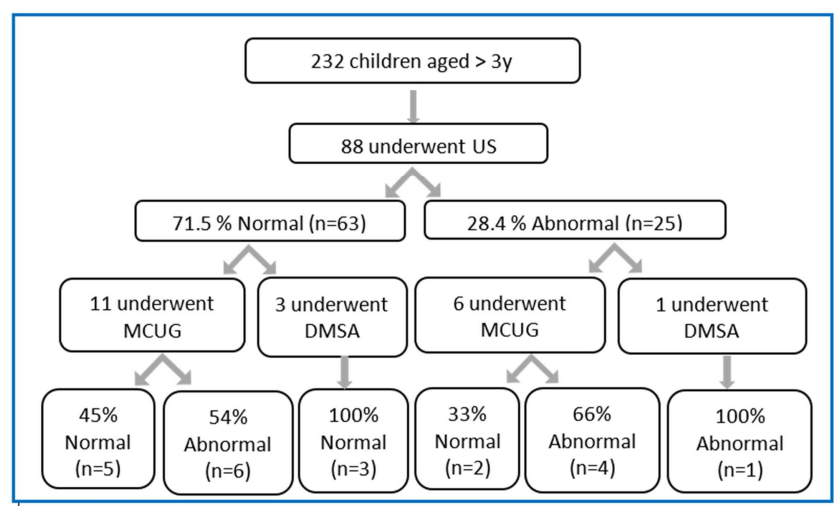

Figure 6. Patients flow chart of the third age group.

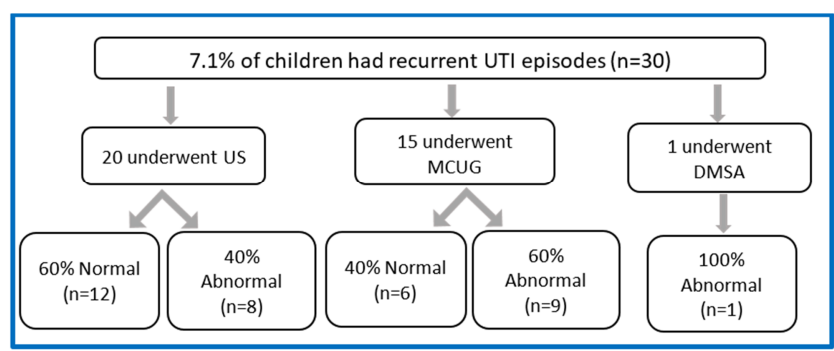

Figure 7. Patients flow chart based on recurrent UTI.

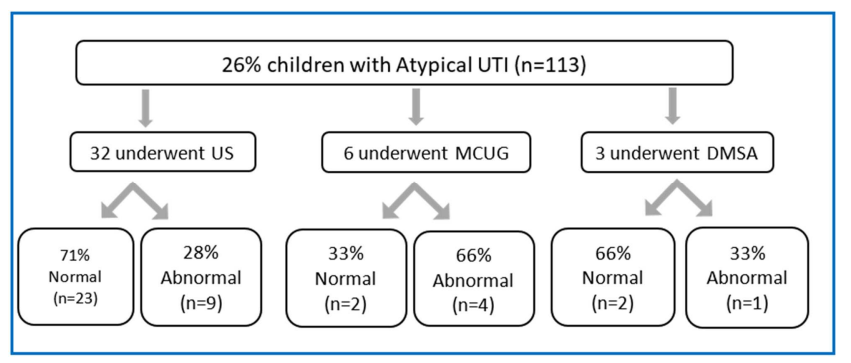

Figure 8. Patients flow chart based on atypical UTI.

Radio-nuclear investigations following UTI in children is continued to be controversial issue. Risks against benefits especially among the younger age group less than 6 months old, the recurrent UTI and the atypical UTI, needs to be considered carefully and may require local guidelines based on proficient research. Risk factors that may predisposes children to complicated recurrent episodes of UTI are renal obstruction, VUR and scarring [9]. In this study, most UTI cases occurred in the third age group (above 3 years old) with female predominance. These findings are compatible with other international figures, where they reported that the male to female UTI ratio is 1:1.5 [10 and 11]. However, a study conducted in Thailand suggested opposing results [12]. The most common uropathogen organism in UTI is previously stated to be Escherichia coli [11], this is also aligned in this study.

KUB US is considered as one of the few safest and affordable investigation used for the overall assessment of the urinary tract [13]. However, KUB ultrasound itself may not be adequate enough as sole test for investigating children following UTI [14 and 15]. This study illustrates, 75\% and $54 \%$ of abnormal MCUG results occurred in patients who had normal KUB US in the second ( 7 months to 3 years old) and third age groups (above 3 years old) respectively. These findings are against the fact that renal US is sufficient to rule out serious anatomical abnormalities or even scars. Hence, this study confirms that KUB US alone is insufficient to assess renal complications. Similar findings were reported in china before [16]. The role of KUB US for managing uncomplicated UTI was questioned by other investigators too [17].

In the youngest age group, $23 \%$ of patients who underwent KUB US had abnormalities. However, it was noted that no further investigations were done. Thus, findings are not enough to ascertain conduction of US, MCUG, or DMSA. However, based on the finding of the older age groups, it is logical to argue that this age group will benefit from assessing DMSA and MCUG. Also, this age group is at more risk of scaring and anatomical abnormalities, hence they need to be investigated right after the first attack of the UTI [4]. The NICE - UK guideline (CG54) (last updated on October 2018) recommends children in this age group with atypical UTI and or abnormal KUB US to undergo, MCUG, and TMP at time of infection and DMSA 4 months later [18].

It is interesting to note that in the second age group (7 months to 3 years old), $3 / 38 \quad(70 \%)$ of children who underwent MCUG with normal US results showed abnormalities and $100 \%(n=4)$ of children who underwent MCUG with abnormal US had VUR. Only one child had abnormal DMSA results despite normal US results. Given these findings, KUB US alone is not enough to exclude complicated UTI even if it is normal hence children aged 7 months to 3 years will need to undergo MCUG and DMSA or MAG3 with post-micturition study regardless of the type of UTI. This study also showed that recurrent and atypical UTI are at high risks of developing complications, therefore children of all ages with recurrent or atypical UTI are recommended to be investigated early. The NICE-UK guidelines suggests to have no investigations carried if the case was typical UTI, and to conduct US, MCUG \& DMSA 4 months later if the case was recurrent or atypical [18]. A low threshold of radio nuclear investigation in this age group $\backslash$ should be considered in the second episode of UTI, also taking into consideration family history and other renal pathology.

In the third age group (above 3 years old), more than 54\% had abnormal MCUG results despite the normal US scans. This is against the claim that KUB US will be able to detect grade 2 VUR [3]. In fact, our findings are consistent with a similar study conducted in China [16]. None of the children in this age group further underwent DMSA. It is therefore, $11.3 \%$ of those older than 3 years with UTI had VUR irrespective of the US outcome. This is certainly a clinically significant value, and should be considered when conducting imaging investigation, especially in this age group. 
Although this study revealed $66.6 \%$ of recurrent cases of UTI occurring in the 3rd age group, a study published in London claims otherwise [15]. The reason may be explained by that more than half the children $(55 \%)$ enrolled in this study fall under the third age group, which gives the assumption of higher rates.

Interestingly higher percentages of US abnormalities were found in males who underwent US (62\%), but higher abnormal DMSA percentages were found in female (100\%). Similar finding was reported previously but with different percentages [7].

It is well known that children with recurrent UTI are at higher risk of scarring and other complications [4 and 5]. In this study $60 \%$ of patients of all ages with recurrent UTI had abnormal MCUG results irrespective of the finding of the KUB US. These finding augment the suggestions of that all the 3 scans should be done for children with recurrent UTI namely KUB US, MCUG (alternative MAG3 with postmicurating study) and DMSA. The NICE-UK guideline recommends likewise [18].

Moreover, these results showed 26\% $(n=113)$ had atypical UTI infections. Out of these, $66 \%$ showed abnormal MCUG, with VUR while 33\% showed abnormal DMSA (renal scaring). The NICE-UK guideline recommends to undergo MCUG and DMSA only if KUB US was abnormal [18].

Findings of this study will help reduce conduction of unnecessary investigations, and help apprise guideline provision efforts to review their guidelines. Although the study was limited in numbers, it reports that despite the normal US results, patients still showed VUR or renal scarring. Hence, this may indicate the need for adjustment and revision of clinical guidelines.

After searching the English literature, this study is the first of its kind in the United Arab Emirates; assessing conduction of radio nuclear investigations in pediatric children. However, a limitation of this research is that conclusions were built on small statistics, which to some extent can be limiting. On the other hand, this study is limited by the lack of documentation details, which are required to reduce bias, such as; if the patient had undergone any of the investigations anywhere else. For generalizability, records that were studied in this research were only limited to one hospital in the Emirate of Dubai, $\mathrm{MCH}$. Considering that $\mathrm{MCH}$ falls under the private sector, this limits external validity, and thus may not be the perfect representative of the UAE population. In view to this limitation, future research may need to consider allocating different hospitals from all around the UAE, including a wide spectrum of governmental and private hospitals. Also, this study did not take into account conduction pattern of US, MCUG, and DMSA. Future researches may want to build conclusions on this criteria, which may give an idea of the sensitivity and selectivity of each investigations.

\section{Conclusion}

These results showed that, MCUG and DMSA scans can turn abnormal irrespective of the KUB US scans results and age groups. However, VUR and renal scarring are predominant in those who presented with atypical and especially with recurrent UTI. It is therefore recommended to keep low threshold for radio nuclear investigation in children with UTI after normal KUB US in young children and those with recurrent and atypical UTI. Other risk factors such as; family history of renal problems, and associated risk factors should be taken in consideration in each case. Conduction of radio- nuclear investigation should be considered during the second UTI episode, rather than waiting for a third episode to fulfil the recurrent UTI criteria.

\section{Disclosure Policy}

The authors declare that there is no conflict of interest regarding the publication of this paper.

\section{Ethical Approval}

This study has been approved by the MBRU-IRB (Mohammad Bin Rashid University - Institutional Review Board) ethical committee and Mediclinic research committee. (MBRU-IRB-SRP2018-034).

\section{Acknowledgements}

Appreciation and sincere gratitude goes to Dr. Tom Loney and Dr. Aida Azar Associate Professors of public health and Epidemiology at the College of Medicine, Mohammed Bin Rashid University of Medicine and Health Sciences (MBRU). Dr. Amar Hassan Khamis Associate professor of Biostatistics and Genetic Epidemiology at the College of Medicine, MBRU for assisting us with the biostatical analysis aspect of the research. Ms. Nesh Ali, microbiologist and Dr. Handan consultant microbiologist at $\mathrm{MCH}$, for allowing and providing us with a list of patients of whom met the inclusion criteria of our study. Appreciation also goes to Mr. Deependra Singh Negi training officer at Mediclinic City Hospital for organizing a training session on the Hospital Information System (HIS).

\section{References}

[1] Alter SJ, Vidwan NK, Sobande PO, Omoloja A, Bennett JS. Common childhood bacterial infections. Current neurology and neuroscience reports. U. S. National Library of Medicine; 2011 Nov; 41 (10): 256-83.

[2] Bleidorn J, Hummers-Pradier E, Schmiemann G, Wiese B, Gagyor I. Recurrent urinary tract infections and complications after symptomatic versus antibiotic treatment: follow-up of a randomised controlled trial. Ger Med Sci. 2016; 14 Doc0.

[3] Uwaezuoke SN. Radiological Investigations of Urinary Tract Infection in. SM Group. 2015. (http://smgebooks.com/urinarytract infections/chapters/UTI-15-03.pdf). 
[4] Pokrajac D, Sefic-Pasic I, Begic A. Vesicoureteral Reflux and Renal Scarring in Infants After the First Febrile Urinary Tract Infection. Med Arch. 2018 Oct; 72 (4): 272-275.

[5] Yılmaz İ, Peru H, Yılmaz FH, Sekmenli T, Çiftçi İ, Kara F Association of vesicoureteral reflux and renal scarring in urinary tract infections. Arch Argent Pediatr. 2018 Aug 1; 116 (4): e542-e547

[6] Rennick, Janet E., et al. "Children's Psychological Responses After Critical Illness and Exposure to Invasive Technology." Journal of Developmental \& Behavioral Pediatrics, vol. 23, no. 3,2002 , pp. $133-144$.

[7] Nasaif MN, Alghamdi AH, Ghamdi JA, Al-Dammas A. The results of different diagnostic imaging studies used in children with urinary tract infection. Sudanese Journal of Paediatrics. Sudan J Paediatr. 2015; 15 (1): 27-36.

[8] Christian MT, McColl JH, MacKenzie JR, Beattie TJ. Risk assessment of renal cortical scarring with urinary tract infection byclinical features and ultrasonography. Arch Dis Child 2000; 82: 376-380.

[9] Becknell B, Schober M, Korbel L, Spencer JD. The Diagnosis, Evaluation and Treatment of Acute and Recurrent Pediatric Urinary Tract Infections. Expert Rev Anti Infect Ther. 2015 Jan; 13 (1): 81-90.

[10] Flores-Mireles AL, Jennifer NW, Michael C, Scott J. Urinary tract infections: epidemiology, mechanisms of infection and treatment options. Nat Rev Microbiol. 2015 May; 13 (5): 269 284.

[11] Wei Tan C, Chlebicki MP. Urinary tract infections in adults. Singapore Med J. 2016 Sep; 57 (9): 485-490.
[12] Supavekin S, Kucivilize K, Hunnangkul S, Sriprapaporn J, Pattaragarn A, Sumboonnanonda A. The relation of vesicoureteral reflux and renal scarring in childhood urinary tract infection. J Med Assoc Thai. 2006 Aug;89 Suppl 2: S417.

[13] Gamss R, Stein MW, Rispoli JM, Cohen HW, Roberts JH, Koenigsberg M, Mazzariol FS, What is the Appropriate Use of Renal Ultrasound in an Inner City Population with New Onset Acute Kidney Injury? J Ultrasound Med. 2015 Sep; 34 (9): $1639-1644$

[14] Biassoni L, Chippington S. Imaging in urinary tract infections: current strategies and new trends. Semin Nucl Med. 2008 Jan; 38 (1): 56-66.

[15] Tewary K, Narchi H. Recurrent urinary tract infections in children: Preventive interventions other than prophylactic antibiotics. World J Methodol. 2015 Jun 26; 5 (2): 13-19.

[16] Vachvanichsanong P, Dissaneewate P, McNeil E. What Did We Find From Imaging Studies in Childhood Urinary Tract Infection and Which Studies Are Mandatory? Urology. 2018 Jan; 111: 176-182.

[17] Zamir G, Sakran W, Horowitz Y, Koren A, Miron D. Urinary tract infection: is there a need for routine renal ultrasonography? Arch Dis Child. 2004 May; 89 (5): 466-8.

[18] Platt C, Larcombe J, Dudley J, McNulty C, Banerjee J. Urinary tract infection Guidline in under 16s: diagnosis and management. Acta Paediat. 2015; Vol 104(6):630-7. 2007. (https://www.nice.org.uk/guidance/cg54). 